\title{
Is Hydroxychloroquine a Safe and Effective Choice for Covid- 19 Prophylaxis
}

\author{
${ }^{1}$ Rodrigo Moisés de Almeida-Leite, ${ }^{2}$ Tais Rondello Bonatti, ${ }^{3}$ Carolina Rodrigues Ponzoni, \\ ${ }^{4}$ Ana Cristina da Cunha Korndorfer and ${ }^{5}$ Solange Bifani Tavares da Silva \\ ${ }^{1}$ Department of Medicine, Faculdade de Medicina de Jundiaí, Brazil \\ ${ }^{2}$ Department of Parasitic Diseases, Universidade Paulista, Brazil \\ ${ }^{3}$ Department of Intensive Care Medicine, Unimed Itatiba, Brazil \\ ${ }^{4}$ Department of Internal Medicine, Faculdade de Medicina de Teresópolis, Brazil \\ ${ }^{5}$ Department of Pediatrics, Faculdade de Medicina de Jundiaí, Brazil
}

Article history

Received: 16-04-2020

Revised: $15-05-2020$

Accepted: 29-07-2020

Corresponding Author:

Rodrigo Moisés de Almeida-

Leite

Department of Medicine,

Faculdade de Medicina de

Jundiaí, Brazil

Email: rodrigomoises@me.com

\begin{abstract}
The authors speculate about the possibility of Hydroxychloroquine as Covid-19 Phrophylaxis in the dose of $400 \mathrm{mg}$ per week. The authors compared the experience of Malaria phrophylaxis in this dose, with acceptable safety profile. Also, we compared in vitro susceptibility of SARS-Cov-2 with that of Plasmodium falciparum. This agent show significant higher potency for the first. Also, the extended Halflife (40-50 days) make it an acceptable choice for prophylaxis. We also cite Brazilian experience with Chloroquinated Salt in the 1950s, its efficacy and feasibility. Lastly, we suggest that clinical trials should be conducted test this hypothesis.
\end{abstract}

Keywords: COVID-19, Pre-exposure Prophylaxis, Hydroxychloroquine
Hydroxychloroquine has been used for more than sixtyfive years in diverse clinical settings around the globe. Current FDA approved uses are for Systemic Lupus Sclerosis, Discoid Lupus and treatment and prevention of malaria. The recommended dose for Malaria prophylaxis is $400 \mathrm{mg}$ per week (Shippey et al., 2018).

Many agents have show in vitro and pre-clinical activity against this virus, including Remdesivir, Chloroquine and Hydroxychloroquine (Wang et al., 2020). The latter has shown more potent antiviral activity against SARS-CoV-2 and a better safety profile when compared with chloroquine. Shippey et al. (2018; Wang et al., 2020) hydroxychloroquine half-maximal Effective Concentration $\left(\mathrm{EC}_{50}\right)$ for SARS-CoV-2 is substantially lower when compared to that of chloroquine - hydroxychloroquine $\mathrm{EC}_{50}=6.14 \mu \mathrm{M}$ and chloroquine: $\mathrm{EC}_{50}=23.90 \mu \mathrm{M}$. Sanders et al. (2020) also, Hydroxychloroquine has show more potent activity against SARS-CoV-2 when compared with activity against susceptible Plasmodium falciparum, expressed by a substantially lower minimum inhibitory concentration (Lim et al., 2009). Additionally, extended half-life of the drug, around 40-50 days 1, promote it as a reasonable alternative for Covid-19 prophylaxis, with better safety-profile and greater potency when compared to chloroquine (Sanders et al., 2020; Lim et al., 2009; Yao et al., 2020; Watson et al., 2018).

Notwithstanding promising pre-clinical evidence, caution should be taken before drawing conclusions about mass-strategy use. The exact mechanism by which Hydroxychloroquine inhibits SARS-CoV-2 in humans is yet unknown and may be different from that of Plasmodium spy (Sanders et al., 2020). For the latter, is accepted to be by acidification of lysosomes, whereas for SARS-CoV-2 is believed to be by endocytosis and membrane fusion prevention, coupled with host immunomodulatory effects (Sanders et al., 2020). This different pharmacodynamics may have implications in the efficacy as a prophylactic and therapeutic drug. Moreover, dose strategies are still obscure. To this date, is proposed that a lower dose of Hydroxychloroquine is adequate for Covid-19 when compared to Malaria. Whether this translates to prophylactic strategy is still unknown (Sanders et al., 2020).

Mass-prophylaxis with chloroquine or hydrocychlorquine has been considered as strategy for Covid-19 mitigation (Spinelli et al., 2020). Specifically in Brazil, Malaria mass prophylaxis with Pinotti Salt (chloroquinated salt) was conducted and found to be feasible and effective in the Amazon region during the 1950s and 1960s (Da Silva and Hochman, 2011). 
To this date, limited evidence is available regarding in vivo efficacy in the treatment and prophylaxis of Covid-19. The scarce literature comprimes mostly of restrospective analysis with demographically distinct groups. One ecological study conducted in France compared mortality time to double among 16 countries with mass use of chloroquine and hydroxychloroquine and compared to demographically similar countries without this strategy. This study show an statistically significant reduction in the $R 2$ from 0.97 to 0.73 with the massstrategy use of anti-malarial drugs (Izoulet, 2020).

Additionally, the use of Hydroxychloroquine does not appear to significantly increase adverse effects, as noted by a recent retrospective analysis. These authors present the outcomes of early hydroxychloroquine associated with Azythromicin in the treatment of the new coronavirus disease. Overall mortality was reported to be $0,7 \%$ and the authors did not find increased likelihood of serious adverse effects (Million et al., 2020).

In light of the seriousness of the ongoing Covid-19 pandemic, effective mitigations strategies are desperately needed. To date, there is scant pre-clinical and limited invivo evidence for the efficacy of antimalarial drugs for Covid-19 phrophylaxis, with Hydroxychloroquine being a preferable choice for greater potency and better safety profile. Although many questions are still to be answered before broad use for these drugs with this purpose, including precise pharmacodynamics and effective dose, current evidence supports the promotion of clinical trials, which may pave way to a new use for this decade-old drug.

\section{Author's Contributions}

All authors equally contributed in this work.

\section{Ethics}

The authors do not have any conflict of interests to declare. All authors have been personally and actively involved in substantive work leading to the manuscript and will hold themselves jointly and individually responsible for its content.

\section{References}

Da Silva, R., \& Hochman, G. (2011). A method called Pinotti: medicated salt, malaria, and international health (1952-1960). Historia, ciencias, saude-Manguinhos, 18(2), 519.
Izoulet, M. (2020). National consumption of antimalarial drugs and COVID-19 deaths dynamics: An ecological study. medRxiv.

Lim, H. S., Im, J. S., Cho, J. Y., Bae, K. S., Klein, T. A., Yeom, J. S., ... \& Park, J. W. (2009). Pharmacokinetics of hydroxychloroquine and its clinical implications in chemoprophylaxis against malaria caused by Plasmodium vivax. Antimicrobial Agents and Chemotherapy, 53(4), 1468-1475.

Million, M., Lagier, J. C., Gautret, P., Colson, P., Fournier, P. E., Amrane, S., ... \& Aubry, C. (2020). Full-length title: Early treatment of COVID-19 patients with hydroxychloroquine and azithromycin: A retrospective analysis of 1061 cases in Marseille, France. Travel medicine and infectious disease, 101738.

Sanders, J. M., Monogue, M. L., Jodlowski, T. Z., \& Cutrell, J. B. (2020). Pharmacologic treatments for coronavirus disease 2019 (COVID-19): a review. Jama, 323(18), 1824-1836.

Shippey, E. A., Wagler, V. D., \& Collamer, A. N. (2018). Hydroxychloroquine: An old drug with new relevance. Cleveland Clinic journal of medicine, 85(6), 459-467.

Spinelli, F. R., Ceccarelli, F., Di Franco, M., \& Conti, F. (2020). To consider or not antimalarials as a prophylactic intervention in the SARS-CoV-2 (Covid-19) pandemic. Annals of the rheumatic diseases, 79(5), 666-667.

Wang, M., Cao, R., Zhang, L., Yang, X., Liu, J., Xu, M., ... \& Xiao, G. (2020). Remdesivir and chloroquine effectively inhibit the recently emerged novel coronavirus (2019-nCoV) in vitro. Cell research, 30(3), 269-271.

Watson, J., Chu, C. S., Tarning, J., \& White, N. J. (2018). Characterizing blood-stage antimalarial drug MIC values in vivo using reinfection patterns. Antimicrobial Agents and Chemotherapy, 62(7).

Yao, X., Ye, F., Zhang, M., Cui, C., Huang, B., Niu, P., ... \& Zhan, S. (2020). In vitro antiviral activity and projection of optimized dosing design of hydroxychloroquine for the treatment of severe acute respiratory syndrome coronavirus 2 (SARSCoV-2). Clinical Infectious Diseases. 